\title{
MEDI3617, a human anti-Angiopoietin 2 monoclonal antibody, inhibits angiogenesis and tumor growth in human tumor xenograft models
}

\author{
CHING CHING LEOW ${ }^{1}$, KAREN COFFMAN ${ }^{1}$, IVAN INIGO ${ }^{1}$, SHANNON BREEN ${ }^{1}$, \\ MEGGAN CZAPIGA $^{2}$, SERGUEI SOUKHAREV ${ }^{2}$, NEILL GINGLES ${ }^{4}$, NORMAN PETERSON ${ }^{2}$, \\ CHRISTINE FAZENBAKER ${ }^{1}$, ROB WOODS ${ }^{3}$, BAHIJA JALLAL ${ }^{1-3}$, SALLY-ANN RICKETTS ${ }^{4}$, \\ THERESA LAVALLEE ${ }^{2}$, STEVE COATS $^{1}$ and YONG CHANG ${ }^{1}$ \\ ${ }^{1}$ Department of Oncology, ${ }^{2}$ Translational Sciences, ${ }^{3}$ Antibody and Protein Engineering, MedImmune, \\ Gaithersburg, MD 20878, USA; ${ }^{4}$ Imaging, Personalised Healthcare and Biomarkers, \\ AstraZeneca, Alderly Park, Macclesfield, SK10 4TG, UK
}

Received October 21, 2011; Accepted December 2, 2011

DOI: $10.3892 /$ ijo.2012.1366

\begin{abstract}
Angiopoietin 2 (Ang2) is an important regulator of angiogenesis, blood vessel maturation and integrity of the vascular endothelium. The correlation between the dynamic expression of Ang2 in tumors with regions of high angiogenic activity and a poor prognosis in many tumor types makes Ang2 an ideal drug target. We have generated MEDI3617, a human anti-Ang 2 monoclonal antibody that neutralizes Ang2 by preventing its binding to the Tie2 receptor in vitro, and inhibits angiogenesis and tumor growth in vivo. Treatment of mice with MEDI3617 resulted in inhibition of angiogenesis in several mouse models including: FGF2-induced angiogenesis in a basement extract plug model, tumor and retinal angiogenesis. In xenograft tumor models, treatment with MEDI3617 resulted in a reduction in tumor angiogenesis and an increase in tumor hypoxia. The administration of MEDI3617 as a single agent to mice bearing human tumor xenografts resulted in tumor growth inhibition against a broad spectrum of tumor types. Combining MEDI3617 with chemotherapy or bevacizumab resulted in a delay in tumor growth and no body weight loss was observed in the combination groups. These results, combined with pharmacodynamic studies, demonstrate that treatment of tumor-bearing mice with MEDI3617 significantly inhibited tumor growth as a single agent by blocking tumor angiogenesis. Together, these data show that MEDI3617 is a robust antiangiogenic agent and support the clinical evaluation and biomarker development of MEDI3617 in cancer patients.
\end{abstract}

Correspondence to: Dr Ching Ching Leow, Department of Oncology, MedImmune, One MedImmune Way, Gaithersburg, MD 20878, USA

E-mail: leowc@medimmune.com

Key words: Ang2, angiogenesis, xenograft, hypoxia

\section{Introduction}

Angiopoietins and their receptor, Tie2, play important roles in physiological vascular growth and maintenance as well as pathological angiogenesis. Of the four known angiopoietin ligands (Ang1, Ang2, Ang3 and Ang4), Ang1 and Ang2 are better characterized ligands of the Tie2 receptor (1). Although there is approximately a $60 \%$ sequence homology between Ang1 and Ang2, they have distinct functional roles with the role of Ang2 being highly context dependent $(1,2)$. Binding of Ang2 to the Tie2 receptor has been shown to result in dissociation of pericytes from the vascular endothelium of blood vessels, thereby providing a permissive environment for stimulation of the endothelial cells by pro-angiogenic factors such as VEGF and FGF (2,3-5). Studies have shown that Ang1 is important in maintaining vessel stability during the quiescent, mature state of angiogenesis, whereas Ang2 is required for vascular remodeling. Ang2 expression is elevated in the context of active angiogenesis, including pathologic angiogenesis associated with tumorigenesis. Ang2 is stored and released by WeibelPalade bodies in endothelial cells (6). The expression of Ang2 has since been reported to be dynamic, primarily upregulated in regions of either hypoxia (7) or regions undergoing vascular remodeling (8-11).

Evidence for elevated Ang2 levels has been associated with diverse tumor types including melanoma, breast, colon, hepatocellular, renal cell and ovarian carcinoma (1,2,12-15). In contrast, given the role of Ang1 in maintaining vascular stability, Ang1 expression is constitutively expressed in adults $(2,14)$. Several groups have reported low circulating Ang1/Ang2 ratios correlate with either poor survival or more advanced disease (16-18). Tie2 expression is primarily localized to endothelial cells, hematopoietic cells and in certain types of tumor cells such as Kaposi sarcoma and melanoma (19-22). Of note, there also exists a subpopulation of Tie2expressing monocytes that has been reported to possess angiogenic activity associated with the recruitment of tumorassociated macrophages, which are recruited to disease sites 
by Ang2 expression (23), suggesting a role for Ang2 in the tumor microenvironment.

Given the role Ang2 plays in pathological angiogenesis and inflammation, it is an attractive drug target for cancer. In this current study, we describe the characterization of MEDI3617, a human $\operatorname{IgG} 1 \kappa$ monoclonal antibody directed against human Ang2. MEDI3617 was generated using the Xenomouse ${ }^{\circledR}$ technology and is selective for inhibition of recombinant human Ang2 (rhuAng2) binding and phosphorylation of the human Tie2 (huTie2) receptor. Indeed, in tumor xenograft models, treatment of mice with MEDI3617 as a single agent resulted in broad antitumor activity through modulation of tumor angiogenesis. Delayed tumor regrowth was achieved when MEDI3617 was combined with either chemotherapy or bevacizumab. We were able to demonstrate the functional effect on angiogenesis through a multi-modality approach using novel techniques such as fluorescence molecular tomography (FMT) imaging and microCT imaging of vascular casts. Of note, we also observed effects on size of vessel calibers and significant elevation of hypoxia in MEDI3617-treated tumors.

\section{Materials and methods}

Cell lines. Colo205 (human colorectal carcinoma; CCL-222), A498 (human renal cell carcinoma; HTB-44), 786-0 (human renal cell carcinoma; CRL-932), PLCPRF/5 (human hepatocellular carcinoma; CRL-8024), Calu6 (human lung carcinoma; HTB-56), LoVo (human colorectal carcinoma; CRL-229) and HeyA8 (human ovarian carcinoma) cells were obtained from the American Type Culture Collection (ATCC, Manassas, VA). All cell lines were authenticated by DNA profiling for 9 markers in April 2011 (RADIL). Colo205 and 786-0 cells were cultured in RPMI-1640 media containing $10 \%$ fetal bovine serum (FBS), A498 and PLCPRF/5 cells in Eagle's minimum essential medium (EMEM) with 10\% FBS (Invitrogen, Carlsbad, CA) and HeyA8 in DMEM 10\% FBS (Invitrogen). Hek293 cells were transfected with full-length human Tie2 and maintained in DMEM supplemented with 10\% FBS and $2 \mu \mathrm{g} / \mathrm{ml}$ puromycin (Invitrogen). All cell lines were cultured in humidified incubators at $37^{\circ} \mathrm{C}$ and $5 \% \mathrm{CO}_{2}$.

Ang2-Tie2 competitive binding ELISA. Maxisorp plates (Nunc, Rochester, NY) were coated with $4 \mu \mathrm{g} / \mathrm{ml}$ human Tie2-Fc (R\&D Systems, Minneapolis, MN), blocked with $0.5 \%$ BSA $/ 0.1 \%$ Tween-20/PBS buffer, and then washed with $0.05 \%$ Tween-20/PBS. MEDI3617 ranging from 0.4 to $6.6 \mathrm{pM}$ in PBS was added to wells followed by $200 \mathrm{ng} / \mathrm{ml}$ biotinylated huAng2 (R\&D Systems). Plates were washed, incubated with 1:5000 Streptavidin HRP (Pierce, Rockford, IL) and developed with TMB substrate (3, 3', 5, 5'-tetramethylbenzidine) solution (KPL, Gaithersburg, MD). Reaction was stopped and read at OD $450 \mathrm{~nm}$ using a Molecular Devices VMAX plate reader (Sunnyvale, CA).

pTie2 immunoassay. Plates were seeded with $1 \times 10^{6}$ Hek293-Tie2 cells in growth media and incubated overnight $\left(37^{\circ} \mathrm{C}, 5 \% \mathrm{CO}_{2}\right)$. MEDI3617 ranging from $0.02 \mathrm{nM}$ to $133.3 \mathrm{nM}$ were incubated with $2 \mu \mathrm{g} / \mathrm{ml}$ of recombinant Ang2 (R\&D Systems) at a 1:1 ratio. Cells were lysed with RIPA buffer (Boston BioProducts, Ashland, MA) and total protein was quantitated using the bicinchoninic acid assay (BCA) protocol (Pierce).

Plates (MesoScale Discovery, Rockville, MD) were coated with $2 \mu \mathrm{g} / \mathrm{ml}$ anti-human Tie2 antibody (Abcam, Cambridge, MA), blocked (1X TBS, 3\% MSD blocker A, 0.05\% Tween-20) and $10 \mu \mathrm{g}$ of sample lysate was added to each well in duplicates and incubated. Anti-pTie2 antibody $(1 \mu \mathrm{g} / \mathrm{ml})(\mathrm{R} \& \mathrm{D}$ Systems) was added to each well and incubated with $0.5 \mu \mathrm{g} / \mathrm{ml}$ sulfo-tag goat anti-rabbit antibody (MesoScale Discovery) and quantitated with a MesoScale SI6000 plate imager (MesoScale Discovery).

Immunoprecipitation and western blot analysis for total and phosphorylated Tie 2 . Protein samples $(80 \mu \mathrm{g})$ were immunoprecipitated with Tie2 monoclonal antibody (Ab33; Cell Signaling, Danvers, MA) and protein A/G sepharose beads (Pierce). SDS-PAGE was performed with 4-12\% bis-tris gels and phosphorylated and total Tie 2 were detected with anti-phosphotyrosine monoclonal antibody (4G10; Millipore, Billerica, MA) and total Tie2 antibody (Ab33; Cell Signaling).

In vivo studies with subcutaneous tumor xenografts. Female athymic nu/nu mice (Harlan, Indianapolis, IN) were implanted subcutaneously (s.c.) with $3 \times 10^{6}$ Colo 205 or $5 \times 10^{6}$ LoVo or $5 \times 10^{6}$ PLCPRF/5 cells or $5 \times 10^{6}$ cells HeyA8 (50\% Matrigel) or $10 \times 10^{6}$ A498 (50\% Matrigel) cells into the right flank. For the 786-0 model, $3 \mathrm{~mm}^{3}$ size tumor fragments were implanted into right flank. Efficacy was measured as percent tumor growth inhibition (TGI) relative to vehicle treated group. Error bars were calculated as standard error of means and two-way ANOVA was used for statistical analyses ( $\mathrm{n}=10 \mathrm{mice} /$ group). All studies performed on mice in these studies were approved by the MedImmune Institutional Animal Care and Use Committee (IACUC). MedImmune is an Association for Animal Acrreditation of Laboratory Animal Care (AAALAC) accredited facility.

Basement membrane extract (BME) plug assay. Cultrex (Trevigen, Gaithersburg, MD) with or without $1 \mu \mathrm{g} / \mathrm{ml}$ fibroblast growth factor (FGF2) (R\&D Systems) was implanted subcutaneously into female athymic nu/nu mice (Harlan) (day 1). MEDI3617 was administered intraperitoneally (i.p.) at 1,10 or $20 \mathrm{mg} / \mathrm{kg}$ ( $\mathrm{n}=12$ per group) on days 1,4 and 8 of implantation. On day 11, FITC-dextran (Research Organics, Cleveland, $\mathrm{OH}$ ) was administered intravenously and plugs were harvested. Plugs from 6 mice were placed in $10 \%$ neutral buffered formalin (NBF) (VWR, West Chester, PA) and stained with hematoxylin and eosin. Plugs from another 6 mice were homogenized for FITC-dextran content analysis in triplicates and fluorescence measured using EnVision Multilabel Plate Reader (Perkin Elmer, Waltham, MA). Functional vessels were quantitated in relative luminescence units. Results were normalized against BME/PBS plugs. Statistical analyses using Proc GLM in $\mathrm{SAS}^{\circledR}$, and Tukey-Kramer method was used for pair-wise comparison.

Retinal angiogenesis study. CD1 mouse pups (Harlan) were dosed i.p. with $0.3,1.0$ or $10 \mathrm{mg} / \mathrm{kg}$ MEDI3617 ( $\mathrm{n}=3$ in untreated and each treatment group) on days P1 (day of birth), P3, and P5. On P8, pups were perfused with $12.5 \mathrm{mg} / \mathrm{ml}$ FITC-dextran 
A

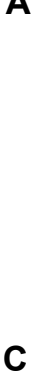

C

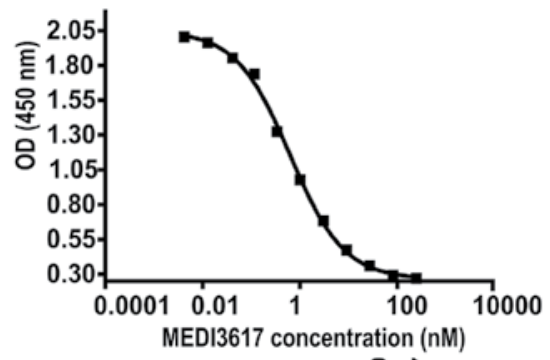

$\sum$ ก

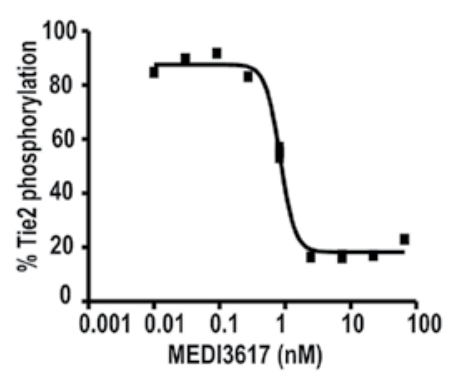

D

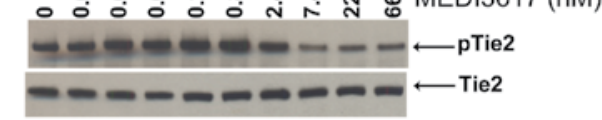

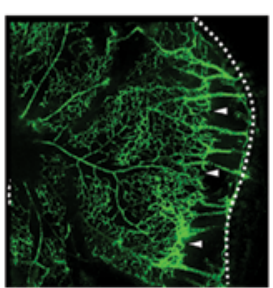

Untreated

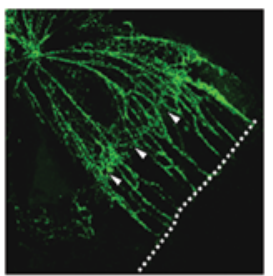

$1 \mathrm{mg} / \mathrm{kg}$ MED|3617

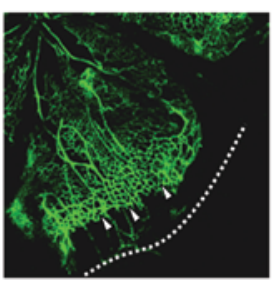

$0.3 \mathrm{mg} / \mathrm{kg}$ MEDI3617

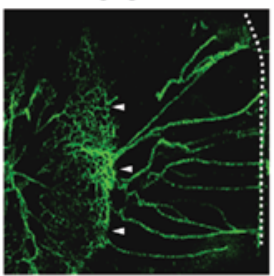

10 mg/kg MEDD3617

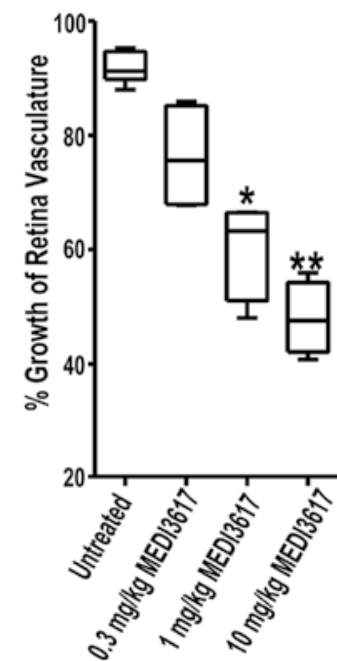

Figure 1. MEDI3617 selectively inhibits rhuAng2 binding to and rhuAng2-induced phosphorylation of Tie2, and inhibits vessel remodeling. (A) MEDI3617 blocked rhuAng2 binding to Tie2 in a rhuAng2-Tie2 competitive binding ELISA. (B) MEDI3617 effectively blocked rhuAng2-induced phosphorylation of Tie2 receptor. (C) Suppression of pTie2 was confirmed by western blot analysis. (D) MEDI3617 was evaluated in an in vivo model of neonatal retinal angiogenesis. Retinal vasculature from $10 \mathrm{mg} / \mathrm{kg}$ MEDI3617 group showed the most significant response of shortening of vessels (arrows, inset). (E) Quantitation of distance migrated as a percentage of maximum distance migrated (white arrow in D) and periphery of retina (white dotted line in D). Significant inhibition of retinal vasculature in the $1 \mathrm{mg} / \mathrm{kg}$ MEDI3617 $(\mathrm{p}<0.05)$ and $10 \mathrm{mg} / \mathrm{kg}$ MEDI3617 ( $\mathrm{p}=0.015)$ in comparison to the untreated group.

(Sigma, St. Louis, MO) through the left ventricle. Eyes were fixed in $10 \% \mathrm{NBF}$, retinas were flat-mounted and imaged with fluorescence microscopy. Percent growth of retinal vasculature was calculated as (distance migrated from optic nerve/distance of edge of retina from optic nerve x100). Three retinas were examined from each group, two measurements were taken from each retina flap and one-way ANOVA was used for statistical analyses.

Vascular casting and micro-computed tomography $(C T)$ imaging. Mice bearing Colo205 tumor xenografts received $1000 \mathrm{U} / \mathrm{ml}$ heparin i.p. and $17 \mathrm{ml}$ of microfil MV-122 (Flow Tech, Carver, MA) was perfused at a rate of $2 \mathrm{ml} / \mathrm{minute}$. The cast were dehydrated from 25 to $100 \%$ ethanol gradient and cleared in methyl salicylate. Brightfield images were captured using a Nikon Eclipse 50i microscope. CT images (Inveon CT module, Siemens Medical Solutions USA, Inc, Knoxville, TN) were acquired at $80 \mathrm{kV}$ with a current of $500 \mu \mathrm{A}$ at a nominal resolution of $18 \mu \mathrm{m}$. CT images were reconstructed using COBRA-EXXIM software using a downsample factor of 1 and a Shepp-Logan backprojection filter method.

Hypoxia detection in Colo205 xenografts. Female athymic $\mathrm{nu} / \mathrm{nu}$ mice (Harlan) bearing $200 \mathrm{~mm}^{3}$ Colo205 tumors were injected intravenously (i.v.) with $60 \mathrm{mg} / \mathrm{kg}$ Hypoxyprobe (HPI, Burlington, MA). Immunostaining with anti-hypoxyprobe antibody (HPI) was performed on acetone-fixed, frozen tissue sections. Hypoxic region was quantitated using the Aperio ${ }^{\mathrm{TM}}$ positive pixel count algorithm (Aperio, Vista, CA) and one-way ANOVA used for statistical analyses.

Fluorescent molecular tomography imaging. Fluorescent Molecular Tomography (FMT; VisEn Medical, Woburn, MA) imaging was performed and treatment was initiated on mice once their tumors reached $\sim 450 \mathrm{~mm}^{3}$. Mice received either no treatment $(n=4)$ or $10 \mathrm{mg} / \mathrm{kg}$ MEDI3617-treated $(\mathrm{n}=4)$ groups on day 1 and 5 after baseline images were obtained. Mice were scanned in the FMT on day 0 (baseline) and day 8 as follows: mice were intravenously injected with 2 nmol AngioSense 750 (blood pooling agent) (VisEn Medical) and scanning was performed at 745/780 $\mathrm{nm}$ (excitation/ emission). Mann Whitney statistical analysis was conducted to demonstrate statistical significance in this study.

\section{Results}

MEDI3617 inhibits rhuAng2 binding and phosphorylation

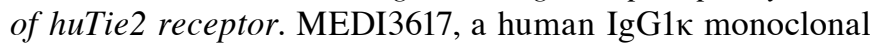


A

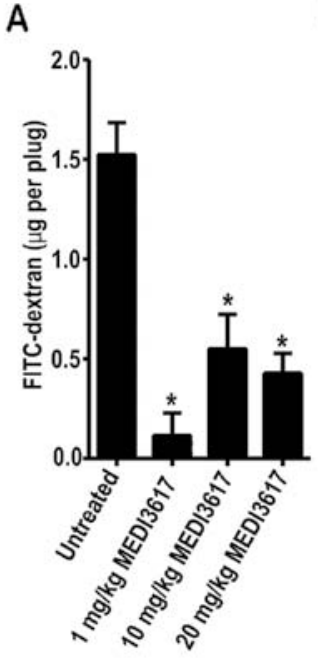

$\mathrm{B}$

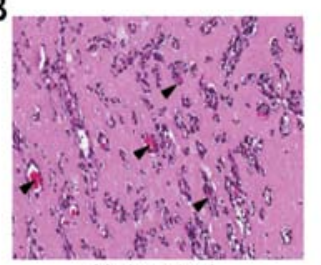

Untreated

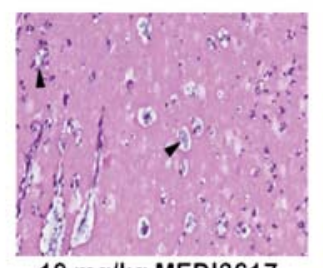

$10 \mathrm{mg} / \mathrm{kg}$ MEDI3617

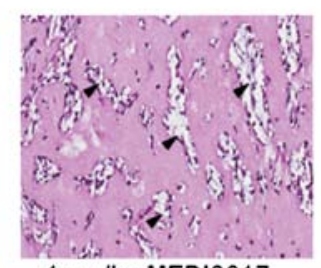

$1 \mathrm{mg} / \mathrm{kg}$ MEDI3617

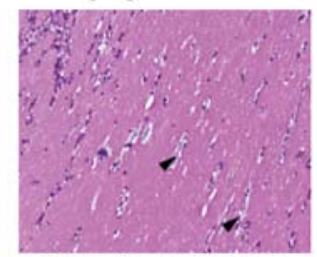

$20 \mathrm{mg} / \mathrm{kg}$ MEDI 3617

Figure 2. MEDI3617 inhibits blood vessel formation in a BME plug assay. (A) Quantitation of FITC-dextran showed significant reduction of FITC-dextran content in plugs from MEDI3617 treated animals compared to untreated control. (B) Sections of BME/FGF2 plugs from untreated or MEDI3617 treated mice, stained with hematoxylin and eosin to visualize vessels. Note a striking reduction of vessels in the $10 \mathrm{mg} / \mathrm{kg}$ and $20 \mathrm{mg} / \mathrm{kg}$ MEDI3617 groups compared to untreated. Arrowheads denote functional and nonfunctional vessels with and without red blood cells, respectively.

antibody that recognizes the fibrinogen binding domain of human Ang2 was generated using the Xenomouse technology, with equilibrium dissociation constant $\left(\mathrm{K}_{\mathrm{D}}\right)$ for the binding of MEDI3617 to rhuAng2 determined to be $42 \mathrm{pM}$. We demonstrated using a competitive ligand-binding ELISA assay that MEDI3617 selectively inhibits binding of rhuAng2 to the huTie2 receptor with an $\mathrm{EC}_{50}$ of $0.510 \mathrm{nM}\left(\mathrm{R}^{2}=0.999\right.$; Fig. 1A) but does not compete with rhuAng1, rhuAng3 or rhuAng4 (data not shown). As shown in Fig. 1B and C, MEDI3617 effectively blocked the rhuAng2-induced phosphorylation of the Tie2 receptor with an $\mathrm{IC}_{50}$ value of $0.85 \mathrm{nM}\left(\mathrm{R}^{2}=0.992\right)$ using HEK293 cells overexpressing human Tie2. On the other hand, MEDI3617 was ineffective in blocking rhuAng1-induced phosphorylation of the Tie2 receptor (data not shown).

MEDI3617 inhibits retinal angiogenesis. MEDI3617, which is mouse cross-reactive, was evaluated in vivo for its ability to inhibit neonatal retinal angiogenesis, a developmental process previously demonstrated to be dependent on Ang2 expression $(9,24)$ and, thus, not to be generalized with mature vessel formation in adults. As shown in Fig. 1D, retinal vasculature from both untreated and $0.3 \mathrm{mg} / \mathrm{kg}$ MEDI3617 treated pups migrated from the optic nerve to the proximity of the retina edge (dotted lines). Both the 1 and $10 \mathrm{mg} / \mathrm{kg}$ MEDI3617 treated groups, respectively, demonstrated inhibition of retinal angiogenesis, where $1 \mathrm{mg} / \mathrm{kg}$ MEDI3617 ( $\mathrm{p}<0.05)$ and $10 \mathrm{mg} / \mathrm{kg}$ MEDI3617 $(\mathrm{p}=0.0015)$ resulted in similar significant inhibition in comparison to the untreated group (Fig. 1D and E).

MEDI3617 inhibits FGF2-induced angiogenesis in basement membrane plug models. The anti-angiogenic activity of 1,10 or $20 \mathrm{mg} / \mathrm{kg}$ MEDI3617 in blocking FGF2-induced angiogenesis was evaluated in BME plugs implanted into athymic nude mice. A significant reduction in the amount of FITC-dextran content within the BME plugs was detected in the $1 \mathrm{mg} / \mathrm{kg}(\mathrm{p}=0.0001)$, $10 \mathrm{mg} / \mathrm{kg}(\mathrm{p}=0.0002)$, and $20 \mathrm{mg} / \mathrm{kg}$ MEDI3617 $(\mathrm{p}=0.0001)$ treated groups relative to untreated animals (Fig. 2A).
BME/FGF2 plugs harvested from another set of mice were stained with hematoxylin and eosin in order to visualize the vessels within the plugs. The non-cellular BME component appears more eosinophilic and the only hematoxylin-positive nuclei of neoangiogenic vessels within the plugs stained purple. As shown in Fig. 2B, sections from untreated BME/FGF2 plugs appeared dense with vessels containing red blood cells (arrow head), indicating that these are functional vessels that would have been detected through the FITC-dextran perfusion described in Fig. 2A. On the other hand, it was interesting to note that mice treated with 1,10 or $20 \mathrm{mg} / \mathrm{kg}$ MEDI3617, respectively, had vessels devoid of red blood cells (arrow head), which could explain the decreased FITC-dextran perfusion. Overall, plugs evaluated from mice treated with either 10 or $20 \mathrm{mg} / \mathrm{kg}$ MEDI3617 contain fewer vessels (Fig. 2B).

MEDI3617 results in tumor growth inhibition in a broad range of tumor xenograft models. Treatment of mice with MEDI3617 results in varying degrees of tumor growth inhibition (TGI), ranging from 45 to $85 \%$, in a broad spectrum of tumor xenograft models including colorectal cancer (LoVo, Colo205, Fig. 3A and E), renal cell cancer (786-0, Fig. 3B), ovarian cancer (HeyA8, Fig. 3C) and hepatocellular cancer (PLCPRF/5, Fig. 3D). Similar antitumor activity in response to MEDI3617 treatment at 3 and $10 \mathrm{mg} / \mathrm{kg}$ was observed in the LoVo, HeyA8, PLCPRF/5 and Colo205 models (Fig. 3A-E), respectively. The most sensitive model was 786-0 since it showed more potent TGI of $85 \%(\mathrm{p}<0.001)$ and $70 \%(\mathrm{p}<0.001)$ at 1 and $10 \mathrm{mg} / \mathrm{kg}$ MEDI3617 doses, respectively. In Fig. 3E, while the Colo205 tumors continued to progress through the first two treatments with 1,3 and $10 \mathrm{mg} / \mathrm{kg}$ MEDI3617, when the mean tumor volume was $\sim 400 \mathrm{~mm}^{3}$ by day 20 , growth of tumors in groups treated with 3 or $10 \mathrm{mg} / \mathrm{kg}$ MEDI3617 had stabilized for the remainder of the study. Based on the results observed in Fig. 3E, we evaluated the effect of MEDI3617 in more established, larger Colo205 tumors, which should be more dependent on a remodeling vasculature than smaller 

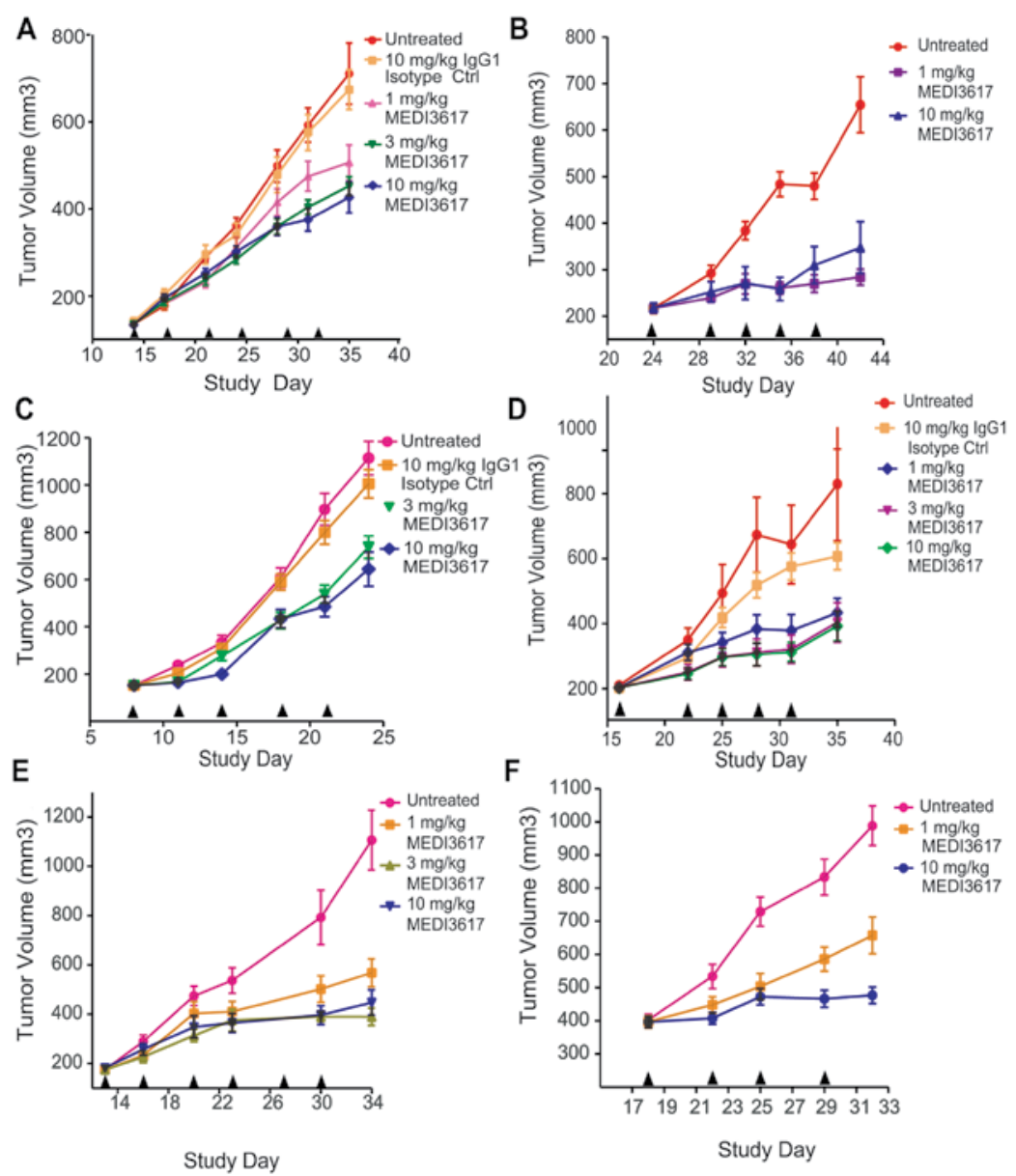

Figure 3. Antitumor activity of MEDI3617 as a single agent in athymic nude mice. Mice bearing (A) LoVo (colon carcinoma) (B) 786-0 (renal cell carcinoma) (C) HeyA8 (ovarian carcinoma) (D) PLCPRF/5 (hepatocellular carcinoma) and (E) Colo205 (colon carcinoma) xenografts were dosed with 1, 3 or $10 \mathrm{mg} / \mathrm{kg}$ MEDI3617, i.p. twice per week for 5-6 doses. (F) Mice bearing larger, $400 \mathrm{~mm}^{3}$ Colo205 xenografts were dosed with 1 or $10 \mathrm{mg} / \mathrm{kg}$ MEDI3617 twice per week for 4 doses. Note significant tumor growth delay after one dose with $10 \mathrm{mg} / \mathrm{kg}$ MEDI3617 compared to when MEDI3617 was administered in smaller tumors as shown in (E). No body weight loss was observed in any of the groups of animals. (₫) Represents time of dose administration.

Table I. Quantitative parameters calculated from reconstructed micro-CT tumor vascular cast samples.

\begin{tabular}{lccc}
\hline Treatment group & $\begin{array}{c}\text { Vascular volume } \\
\left(\mathrm{mm}^{3}\right)\end{array}$ & $\begin{array}{c}\text { Total tumor volume } \\
\left(\mathrm{mm}^{3}\right)\end{array}$ & $\begin{array}{c}\text { Vascular volume fraction } \\
(\%)\end{array}$ \\
\hline Untreated Colo205 tumor \#1 & 20.2 & 350.9 & 5.8 \\
Untreated Colo205 tumor \#2 & 25.3 & 280.0 & 9.0 \\
$10 \mathrm{mg} / \mathrm{kg}$ MEDI3617 treated Colo205 tumor \#1 & 7.4 & 253.1 & 2.9 \\
$10 \mathrm{mg} / \mathrm{kg}$ MEDI3617 treated Colo205 tumor \#2 & 9.4 & 251.0 & 3.7 \\
\hline
\end{tabular}

Parameters calculated by segmentation of observable tumor volume and standard thresholded vasculature.

tumors. As shown in Fig. 3F, treatment with MEDI3617 at a dose of $10 \mathrm{mg} / \mathrm{kg}$ resulted in tumor stasis. A dose-dependent TGI was observed between 1 and $10 \mathrm{mg} / \mathrm{kg}$ MEDI3617, which resulted in a TGI of $56 \%(\mathrm{p}<0.0001)$ and $86 \%(\mathrm{p}<0.0001)$, respectively (Fig. 3F).

MEDI3617 inhibits angiogenesis through modulation of vessel lumen area within tumor xenografts. To further characterize the pharmacodynamic effect of MEDI3617 on tumor vasculature, size-matched, Calu6 tumor xenografts were dosed (i.p. twice) with either 1 or $10 \mathrm{mg} / \mathrm{kg}$ MEDI3617 and harvested $24 \mathrm{~h}$ later. The Calu6 model was chosen for study because the vasculature appears more homogeneously distributed throughout the tumor. Immunostaining with anti-CD31 antibody revealed significant reduction in vessel lumen area. Treatment with $1 \mathrm{mg} / \mathrm{kg}$ and $10 \mathrm{mg} / \mathrm{kg}$ MEDI3617 resulted in 51\% (p=0.016) and $59 \%(\mathrm{p}=0.0041)$ decrease in mean lumen area, respectively (Fig. 4A). In addition, we used a combination of vascular casting technique (Fig. 4B) and microCT imaging (Fig. 4C and Table I), to evaluate the functionality of tumor vessels after treatment 
A

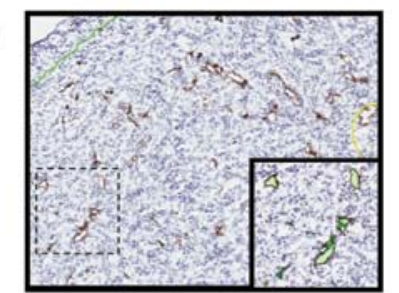

Untreated

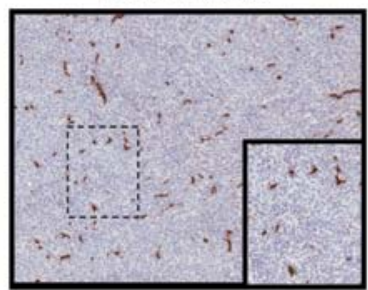

$10 \mathrm{mg} / \mathrm{kg}$ MEDI3617

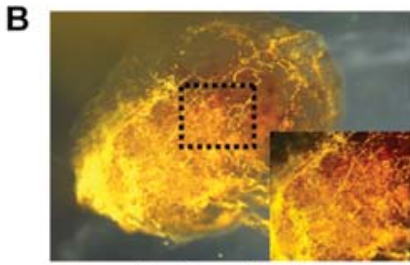

Untreated

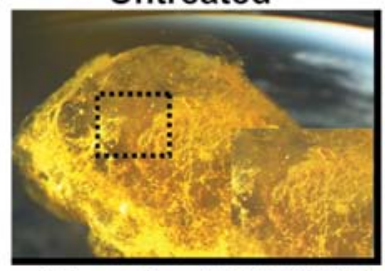

$10 \mathrm{mg} / \mathrm{kg} \mathrm{MEDI3617}$

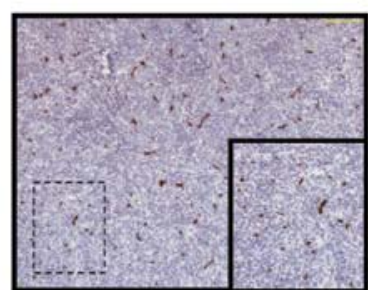

$1 \mathrm{mg} / \mathrm{kg}$ MEDI3617
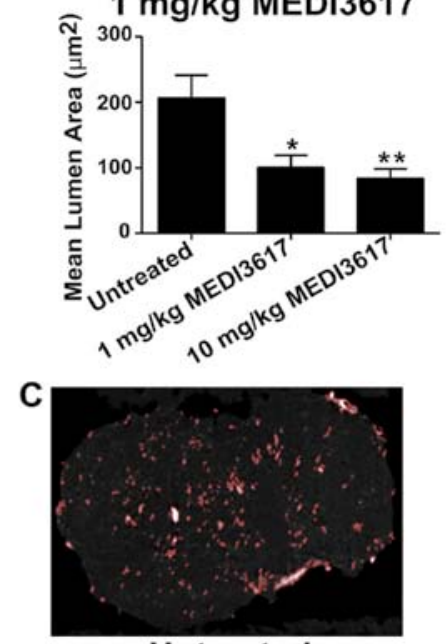

Untreated

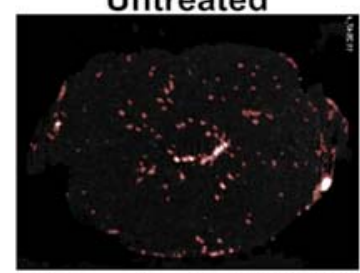

$10 \mathrm{mg} / \mathrm{kg}$ MEDI3617

Figure 4. MEDI3617 activity in vessel remodeling. (A) Functional impact of MEDI3617 on tumor angiogenesis in Calu6 tumor-bearing mice after 2 doses of 1 or $10 \mathrm{mg} / \mathrm{kg} \mathrm{MEDI3617.} \mathrm{Aperio}{ }^{\mathrm{TM}}$ quantitation of CD31 immunostaining revealed a significant reduction in vessel lumen area of $1 \mathrm{mg} / \mathrm{kg}$ MEDI3617 $(\mathrm{p}=0.016)$ and $10 \mathrm{mg} / \mathrm{kg}$ MEDI3617 ( $\mathrm{p}=0.0041$ ) treated groups. (B) Vascular casting was performed for visualization of tumor vasculature in Colo205 xenografts. (C) microCT imaging was used to visualize and quantitate the vasculature within these vascular casts (Table I). Visually, there appeared to be more tumor vessels in untreated samples in comparison to tumors treated with $10 \mathrm{mg} / \mathrm{kg}$ MEDI3617. Red color denotes thresholding the density of the casting material within the tumor, hence relating to the tumor vasculature.

with MEDI3617. In comparison to vascular casts derived from untreated Colo205 xenografts, treatment with $10 \mathrm{mg} / \mathrm{kg}$ MEDI3617 reduced the overall vascularity observed within the tumor, indicative of a reduction of functional vessels (Fig. 4B and $\mathrm{C}$, and Table I).

Given the decrease in vessel lumen area and vessel perfusion, we utilized a noninvasive approach using Fluorescence Molecular Tomography (FMT) to monitor the effect of MEDI3617 on tumor angiogenesis in vivo. Specifically, we delivered AngioSense ${ }^{\circledR}$, a fluorescently labeled $250 \mathrm{kDa}$ macromolecule, intravenously into mice such that functional vessels within Colo205 xenografts could be visualized and quantified. As seen in Fig. 5A, while fluorescence signal of untreated group intensified over time (intense red signal at day 8), the fluorescence signal in the $10 \mathrm{mg} / \mathrm{kg}$ MEDI3617 treated group at day 8 diminished (blue signal) in comparison to baseline images (day 1). The fluorescence signal from each mouse on day 8 is quantitated in Fig. 5B, which demonstrates a significant decrease in the MEDI3617 treated group ( $\mathrm{p}=0.03)$.

In order to understand the functional effect of MEDI3617 on the tumor xenografts, we evaluated the hypoxia status of
MEDI3617-treated Colo205 xenografts. Mice were given two doses of either 1 or $10 \mathrm{mg} / \mathrm{kg}$ MEDI3617 and hypoxia status was analyzed $24 \mathrm{~h}$ after the second dose was administered using Hypoxyprobe $^{\mathrm{TM}}$ (Fig. 5C). An Aperio positive pixel count (PPC) algorithm was used to artificially color (red) and quantitate the hypoxic area that stain positively with anti-hypoxyprobe (Fig. 5C and D). Sections from animals treated with either 1 or $10 \mathrm{mg} / \mathrm{kg}$ MEDI3617 were significantly more hypoxic as compared to untreated animals ( $<<0.05$, Fig. 5D). Consistent with this functional effect of hypoxia within these tumors, we were able to detect significant upregulation of HIF-1 $\alpha(\mathrm{p}<0.0001)$ and VEGF $(\mathrm{p}<0.0001)$ protein in tumor extracts isolated from MEDI3617 treated samples at the end of the treatment period (Table II).

Combination of MEDI3617 with chemotherapy and anti-VEGF inhibits growth of human tumor xenograft models in athymic nude mice. In order to maximize tumor growth inhibition by targeting both tumor vessels and the tumor cells, we evaluated the therapeutic potential of combining MEDI3617 with paclitaxel in the A375 human melanoma xenograft model in athymic nude mice. During the dosing phase, tumor volumes of the vehicle 
A
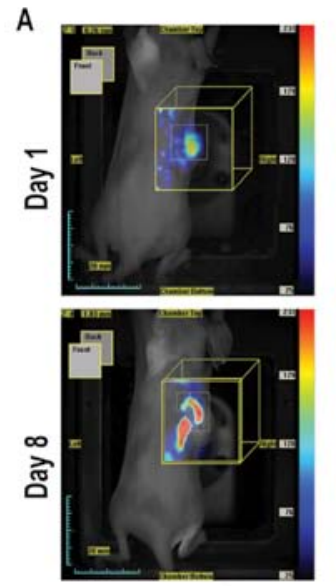

Untreated Group
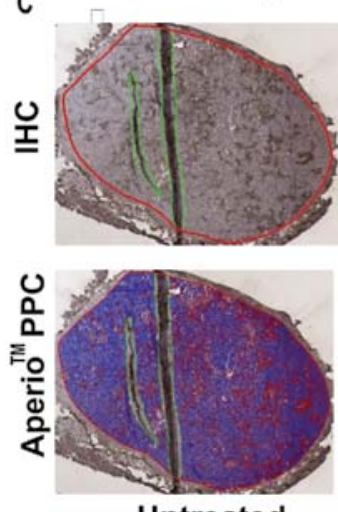

Untreated
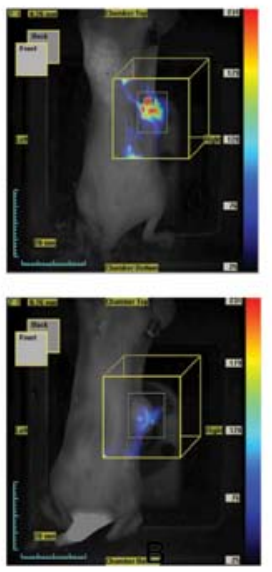

MED3617 Group
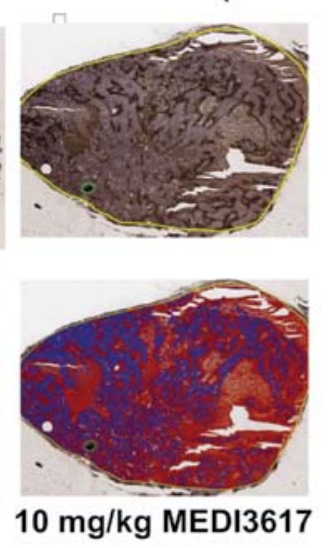

B

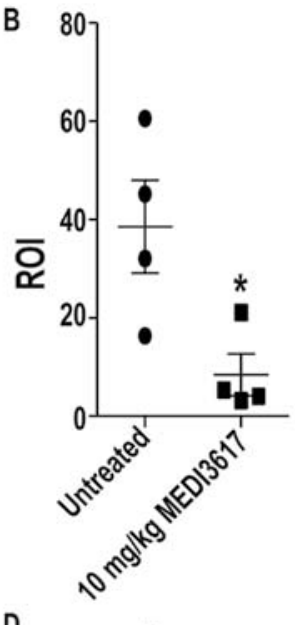

D

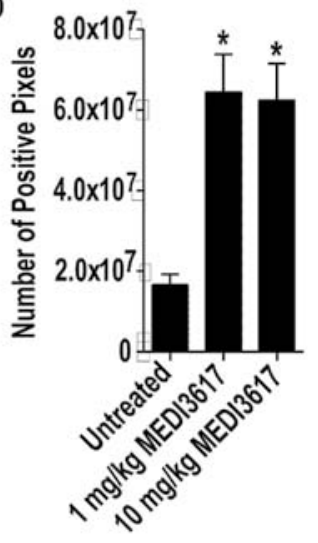

Figure 5. MEDI3617 results in decreased tumor angiogenesis and increased tumor hypoxia. (A) Mice bearing Colo205 colon carcinoma xenografts were injected i.v. with AngioSense750 blood pooling agent and baseline images captured by FMT for all mice (day 1). Mice in the $10 \mathrm{mg} / \mathrm{kg}$ MEDI3617-treated group was dosed on day 1, 5 and final FMT imaging point was collected for all animals on day 8. Images from day 8 showed enhanced AngioSense signal emitted (intense red hue) from untreated tumors $(n=4)$ while AngioSense signal was remarkably diminished (blue hue) in the MEDI3617 treated group ( $\mathrm{n}=4$ ). (B) Quantitation of fluorescent signal from images in A was significantly lower in the MEDI3617 group vs. the untreated group ( $\mathrm{p}=0.03)$ where by signal from the entire tumor was captured and analyzed. (C) Hypoxia status of Colo205 xenografts in mice dosed twice with 1 or $10 \mathrm{mg} / \mathrm{kg}$ MEDI3617 (n=3 each group) and evaluated $24 \mathrm{~h}$ post second dose with Hypoxyprobe ${ }^{\mathrm{TM}}$. Aperio ${ }^{\mathrm{TM}}$ PPC algorithm was used to artificially color and quantitate hypoxic areas (red) where sections stained positively with anti-hypoxyprobe. (D) Sections from animals treated with either $1 \mathrm{or} 10 \mathrm{mg} / \mathrm{kg}$ MEDI3617 were significantly more hypoxic in comparison to untreated tumors $(\mathrm{p}<0.05)$.

Table II. Athymic nude mice bearing Colo205 tumor xenografts were dosed IP for a total of 4 doses and harvested $24 \mathrm{~h}$ after administration of last dose.

\begin{tabular}{lccc}
\hline Treatment & Dose & HIF-1 $\alpha$ & VEGF \\
\hline MEDI3617 & $10 \mathrm{mg} / \mathrm{kg}$, IP, 4 doses & $27 \pm 2 \mathrm{pg} / \mathrm{mg}(\mathrm{p}<0.0001)$ & $145 \pm 11 \mathrm{pg} / \mathrm{mg}(\mathrm{p}<0.0001)$ \\
IgG1 isotype control & $10 \mathrm{mg} / \mathrm{kg}$, IP, 4 doses & $10 \pm 2 \mathrm{pg} / \mathrm{mg}$ & $62 \pm 19 \mathrm{pg} / \mathrm{mg}$
\end{tabular}

Significant upregulation of HIF1 $\alpha$ and VEGF proteins were detected. Comparison was made with tumors treated with IgG1 isotype controls.

and the $\operatorname{IgG1}$ isotype control groups had more than doubled in size whereas treatment with $10 \mathrm{mg} / \mathrm{kg}$ MEDI3617 caused a significant TGI of $27.1 \%(\mathrm{p}<0.0001)$ (Fig. 6A). Treatment with $15 \mathrm{mg} / \mathrm{kg}$ of paclitaxel alone resulted in a robust TGI of $97.2 \%(\mathrm{p}<0.0001)$. Similarly, the combination of paclitaxel with $10 \mathrm{mg} / \mathrm{kg}$ MEDI3617 resulted in a significant TGI of $101.3 \%(\mathrm{p}<0.0001)$. While it did not appear that combining MEDI3617 with paclitaxel enhanced the antitumor efficacy of paclitaxel alone, we observed a $10 \%$ partial regression only in the combination arm of $15 \mathrm{mg} / \mathrm{kg}$ paclitaxel with $10 \mathrm{mg} / \mathrm{kg}$ MEDI3617 (Fig. 6A). During the regrowth phase, combination of $10 \mathrm{mg} / \mathrm{kg}$ MEDI3617 with $15 \mathrm{mg} / \mathrm{kg}$ paclitaxel resulted in significant delayed regrowth $(\mathrm{p}<0.05)$ in comparison to $15 \mathrm{mg} /$ $\mathrm{kg}$ paclitaxel as a single agent (Fig. 6A). Importantly, the combination of MEDI3617 with paclitaxel did not result in body weight loss (data not shown).

Given that it is well-established that the Ang/Tie and VEGF/ VEGFR2 pathways are important in tumor angiogenesis, we 
A
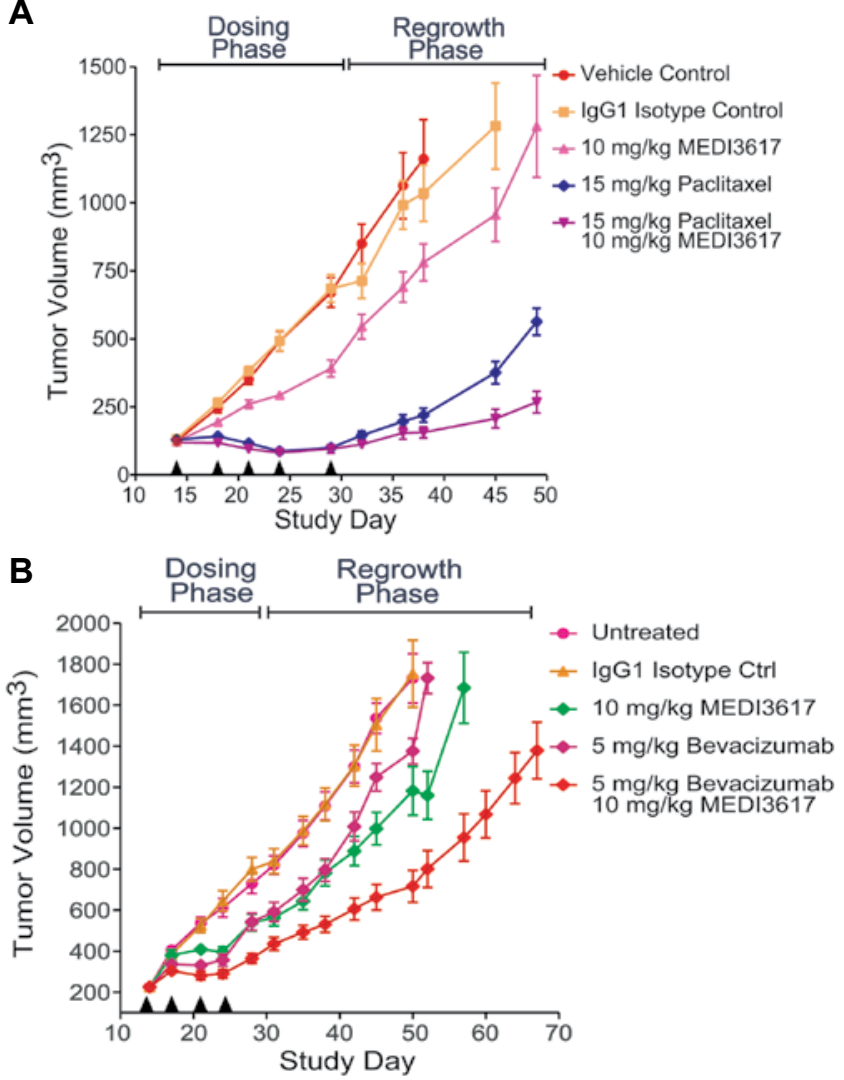

Figure 6. MEDI3617 results in tumor growth delay when combined with paclitaxel or bevacizumab. (A) During the dosing phase, combination treatment of $15 \mathrm{mg} / \mathrm{kg}$ paclitaxel and $10 \mathrm{mg} / \mathrm{kg}$ MEDI3617 resulted in tumor growth inhibition indistinguishable from $15 \mathrm{mg} / \mathrm{kg}$ paclitaxel alone. During regrowth phase, combination of paclitaxel with MEDI3617 showed delayed tumor regrowth in comparison to those treated with paclitaxel alone. (B) During the dosing phase, single agent treatment with either $10 \mathrm{mg} / \mathrm{kg}$ MEDI3617 (2x/wk x 4, IP), $5 \mathrm{mg} / \mathrm{kg}$ bevacizumab (2x/wk x 4, IP) or the combination of both resulted in similar TGI in the HPAC, pancreatic carcinoma xenograft model. During the regrowth phase, combination of MEDI3617 and bevacizumab resulted in delayed tumor regrowth in comparison to those treated with either agent alone.

rationalized that inhibition of both pathways would result in maximal inhibition of tumor vasculature. In addition, as we found that treatment of tumor models with MEDI3617 also results in hypoxia, leading to a significant upregulation of VEGF, these data further emphasize the importance of inhibiting of both Ang2 and VEGF. Since bevacizumab is specific for human VEGF, we could only evaluate such a combination in a xenograft model where the tumor itself was expressing human VEGF. Liang et al have reported that HPAC tumor xenografts express approximately equal amounts of h-VEGF $(116 \pm 30 \mathrm{pg} / \mathrm{mg})$ and $\mathrm{m}-\mathrm{VEGF}(146 \pm 39 \mathrm{pg} / \mathrm{mg})$, and demonstrated that treatment of HPAC tumor-bearing mice with bevacizumab resulted in moderate inhibition of tumor growth (25). As shown in Fig. 6B, during the dosing phase, $10 \mathrm{mg} /$ $\mathrm{kg}$ MEDI3617 administered to mice bearing HPAC tumors resulted in TGI of $26 \%$ and $5 \mathrm{mg} / \mathrm{kg}$ bevacizumab resulted in a TGI of $26 \%$. Combination of $5 \mathrm{mg} / \mathrm{kg}$ bevacizumab with $10 \mathrm{mg} / \mathrm{kg}$ MEDI3617 resulted in TGI of 50\%. During the regrowth phase, the combination of $5 \mathrm{mg} / \mathrm{kg}$ bevacizumab with $10 \mathrm{mg} / \mathrm{kg}$ MEDI3617 resulted in the greatest tumor growth delay than either single agent alone (Fig. 6B). Overall, combination of bevacizumab and MEDI3617 did not result in body weight loss.

\section{Discussion}

Angiopoietin 2 (Ang2) is an important regulator of angiogenesis, blood vessel maturation, and integrity of the vascular endothelium $(1,26)$. The expression of Ang2 in tumors is dynamic and correlates spatially with regions of high angiogenic activity. Increased Ang2 expression has been associated with diverse tumor types which include breast, colon, lung, renal, prostate, and ovarian cancers (13). Increased levels of Ang2 correlated with poor prognosis in non-small cell lung cancer, hepatocellular carcinoma and melanoma $(15,21,27)$. Currently, there are four approved therapies that target the VEGF pathway; anti-VEGF antibody (bevacizumab) and small molecule inhibitors of the pathway (sorafenib, sunitinib, and pazopanib). Although these agents have demonstrated clinical benefit either as single agents or in combination with chemotherapy, there remains a need for further improving patient survival and for better tolerated therapies. The Ang2-Tie2 pathway is another key component of the tumor angiogenesis process, and is an attractive pathway to target to explore clinical benefit and tolerability in pathological angiogenesis such as in cancer patients.

MEDI3617 is an Ang2-selective human IgG1 $\kappa$ anti-Ang2 monoclonal antibody that inhibits rhuAng2 binding and subsequent phosphorylation the Tie2 receptor in vitro. Hackett et al demonstrated that Ang2 expression is upregulated during retinal neovascularization and superficial retinal angiogenesis is incomplete in Ang2-deficient mice (9,24). Indeed, we were able to demonstrate that inhibition of Ang2 via treatment of newborn pups with MEDI3617 resulted in impaired retinal vascular advancement towards the periphery of the retina. In addition, we observed moderate and robust inhibition of the advancing retinal vasculature upon administration of $1 \mathrm{mg} / \mathrm{kg}$ and $10 \mathrm{mg} / \mathrm{kg}$ of MEDI3617, respectively.

We were able to demonstrate varying degrees of tumor growth inhibition in various tumor xenografts and work is ongoing to determine the differences in response observed. Interestingly, we observed that tumor growth inhibition in the Colo205 model was more robust when treatment was initiated in more established tumors, which suggests that larger tumors are more dependent on Ang2. This increased dependency on Ang2 could be fueled by increased hypoxia as growth of tumor cells surpass the vasculature formed from earlier rounds of angiogenesis, thereby triggering Ang2 to destabilize mature vessels formed during earlier rounds of angiogenesis and activating quiescent endothelial cells (reviewed in refs. 1,28). While Nasarre et al (29) concluded from their study that Ang2 is only required for early stages of tumor development, results within this study and Mazzieri et al (30) clearly demonstrated enhanced tumor growth inhibition when treatment was initiated in late stage tumors. More importantly, inhibition of established tumors with MEDI3617 differentiates itself from inhibition of the VEGF pathway, which plays an early role in tumorigenesis by activating the angiogenic switch. In the RIP-Tag model of pancreatic islet cell carcinoma, where progressive neoplastic lesions have been well characterized, neoplastic lesion prior 
to angiogenic switch are highly VEGF-dependent whereas established tumors are less dependent on VEGF (31).

While anti-VEGF treatment displays broad spectrum activity, it is clear that resistance mechanisms can also develop where alternative angiogenesis pathways, such as FGF pathway, are engaged $(32,33)$. We were able to demonstrate MEDI3617mediated inhibition of neoangiogenesis in the BME/FGF2 plug assay resulting in decreased FITC-dextran perfusion into tumor vasculature as well as the lack of both vessels and presence of red blood cells, especially within the core of these plugs. In addition, treatment with MEDI3617 also resulted in moderate tumor growth inhibition in LoVo, a colorectal cancer xenograft model with high expression of FGF2 (Fig. 4A). These results demonstrate that inhibition of Ang2 with MEDI3617 has antiangiogenic effects on FGF2-stimulated angiogenesis as well.

We utilized different modalities to provide evidence of the utility of imaging tumor vasculature as a pharmacodynamic marker for of MEDI3617 mediated Ang2 inhibition. Using FMT imaging, we are able to detect diminished signal emanating from MEDI3617 tumors as opposed to untreated tumors, suggesting that fewer functional vessels were present in the MEDI3617 treated group. These results are consistent with decreased $\mathrm{K}_{\text {trans }}$ values by DCE-MRI in the Phase I studies conducted for AMG-386 and CVX-060 (34,35) and further support this biomarker as a proof of mechanism for Ang2 inhibitors. In addition, we performed microCT imaging of Colo205 vascular casts. From qualitative and quantitative analyses, it was evident from these casts that while untreated tumors were generally highly vascularized, tumors treated with MEDI3617 had an overall reduction in vascularity. The cast data provide further evidence that following admninistration of MEDI3617, there are fewer functional vessels present within treated tumors.

Given the poor vessel perfusion in the MEDI3617 treated groups, we expected that this would lead to enhanced hypoxia within these tumors although this was not previously demonstrated by other studies reporting tumor growth inhibition when interfering with the Ang-Tie2 pathway $(19,36,37)$. In addition we demonstrated that HIF-1 $\alpha$ and VEGF protein expression are significantly upregulated in hypoxic MEDI3617-treated tumors. Taken together these results suggest that MEDI3617 inhibition of Ang-Tie2 pathway results in inhibition of tumor angiogenesis, which in turn leads to an increase in hypoxia within the tumor, ultimately leading to inhibition of tumor growth. More importantly, the observation of a pharmacodynamic effect on tumor vasculature and tumor hypoxia before any decrease in tumor growth is observed suggests that biomarkers for tumor vascularity and/or hypoxia may demonstrate an impact of MEDI3617 treatment prior to any impact on tumor growth measured by RECIST criteria.

Given the data surrounding hypoxia addiction driving tumor invasion and metastasis, it is possible that this is a VEGF-class effect and this theory is not well understood at the present time $(33,38,39)$. In this context, MEDI3617 is also different from VEGF-class agents especially given recent data from Mazzieri et al (30) which demonstrated that anti-Ang2 monoclonal antibody treatment did not result in enhancement of tumor invasion and metastasis. In addition, Holopainen et al also demonstrated that MEDI3617 can inhibit lung and lymph node metastases (40). Another possible mechanism that is currently being investigated is the impact on the integrin pathway since the association between Ang2 and $\alpha 5 \beta 1$ integrin has been previously been shown to affect metastasis (41).

Although we can achieve tumor growth inhibition with MEDI3617 as a single agent, improved tumor growth inhibition can be achieved through targeting of both tumor and vascular components. We evaluated combinations of MEDI3617 (targets tumor vasculature) with chemotherapeutics (targets tumor component) such as paclitaxel and capecitabine (data not shown). These experiments revealed that combination of MEDI3617 with chemotherapy agents resulted in significant tumor growth delay compared to either treatment alone. Improved activity of VEGF-pathway inhibitors with chemotherapy have been partly attributed to vessel normalization $(42,43)$. This is a likely mechanism given that the few functional vessels that remain in MEDI3617-treated groups, combination with chemotherapeutics provides delay tumor regrowth. Since angiogenesis is a process that involves the interplay of multiple signaling pathways, maximal inhibition of angiogenesis can also be achieved by inhibiting both Ang-Tie2 and the VEGF-VEGFR2 pathways. In addition, several groups have reported on the Ang-Tie2 pathway as being one of the compensatory mechanisms around VEGFR inhibitor therapies $(32,44)$. Herein, we demonstrate that inhibition of tumor xenografts with MEDI3617 and bevacizumab can result in further delaying the tumor regrowth. Additional mechanistic studies are currently ongoing to determine the extent of vessel normalization, endothelial cell survival and alternative mechanisms. Two inhibitors, AMG-386 and CVX-060, currently have ongoing clinical trials evaluating the inhibition of these two pathways. Recently, Koh et al reported on DAAP, a double decoy receptor targeting VEGFA/PIGF and Ang1-4 (45) that inhibits tumor angiogenesis, vascular permeability and metastasis. These ongoing clinical trials, effects seen with DAAP that targets VEGF/PIGF and angiopoietin pathways and the findings we report herein support investigating the combination of MEDI3617 and VEGF therapeutics in the clinic. Overall in this study, we provide compelling data around inhibition of vasculogenesis, tumor angiogenesis and tumor growth inhibition. These data provide support for the clinical investigation and further biomarker studies of MEDI3617 in cancer treatment.

\section{Acknowledgements}

We would like to thank Donna Schwartz, Gorazd Drozina and Subash Kahrel for assistance with reviewing and formatting of the manuscript.

\section{References}

1. Augustin HG, Koh GY, Thurston G and Alitalo K: Control of vascular morphogenesis and homeostasis through the angiopoietinTie system. Nat Rev Mol Cell Biol 10: 165-177, 2009.

2. Maisonpierre PC, Suri C, Jones PF, et al: Angiopoietin-2, a natural antagonist for Tie2 that disrupts in vivo angiogenesis. Science 277: 55-60, 1997.

3. Asahara T, Chen D, Takahashi T, et al: Tie2 receptor ligands, angiopoietin-1 and angiopoietin-2, modulate VEGF-induced postnatal neovascularization. Circ Res 83: 233-240, 1998.

4. Inoue M, Hager JH, Ferrara N, Gerber HP and Hanahan D: VEGF-A has a critical, nonredundant role in angiogenic switching and pancreatic beta cell carcinogenesis. Cancer Cell 1: 193-202, 2002.

5. Hanahan D: Signaling vascular morphogenesis and maintenance. Science 277: 48-50, 1997. 
6. Fiedler U, Scharpfenecker M, Koidl S, et al: The Tie-2 ligand angiopoietin-2 is stored in and rapidly released upon stimulation from endothelial cell Weibel-Palade bodies. Blood 103: 4150-4156, 2004.

7. Koga K, Todaka T, Morioka M, et al: Expression of angiopoietin-2 in human glioma cells and its role for angiogenesis. Cancer Res 61: 6248-6254, 2001.

8. Geva E, Ginzinger DG, Zaloudek CJ, Moore DH, Byrne A and Jaffe RB: Human placental vascular development: vasculogenic and angiogenic (branching and nonbranching) transformation is regulated by vascular endothelial growth factor-A, angiopoietin-1, and angiopoietin-2. J Clin Endocrinol Metab 87: 4213-4224, 2002.

9. Hackett SF, Wiegand S, Yancopoulos G and Campochiaro PA: Angiopoietin-2 plays an important role in retinal angiogenesis. J Cell Physiol 192: 182-187, 2002.

10. Mandriota SJ and Pepper MS: Regulation of angiopoietin-2 mRNA levels in bovine microvascular endothelial cells by cytokines and hypoxia. Circ Res 83: 852-859, 1998.

11. Vajkoczy P, Farhadi M, Gaumann A, et al: Microtumor growth initiates angiogenic sprouting with simultaneous expression of VEGF, VEGF receptor-2, and angiopoietin-2. J Clin Invest 109 777-785, 2002.

12. Ahmad SA, Liu W, Jung YD, et al: Differential expression of angiopoietin-1 and angiopoietin-2 in colon carcinoma. A possible mechanism for the initiation of angiogenesis. Cancer 92 1138-1143, 2001.

13. Shim WS, Ho IA and Wong PE: Angiopoietin: a TIE(d) balance in tumor angiogenesis. Mol Cancer Res 5: 655-665, 2007.

14. Tait CR and Jones PF: Angiopoietins in tumours: the angiogenic switch. J Pathol 204: 1-10, 2004.

15. Tanaka S, Mori M, Sakamoto Y, Makuuchi M, Sugimachi K and Wands JR: Biologic significance of angiopoietin-2 expression in human hepatocellular carcinoma. J Clin Invest 103: 341-345, 1999.

16. Anargyrou K, Terpos E, Vassilakopoulos TP, et al: Normalization of the serum angiopoietin-1 to angiopoietin-2 ratio reflects response in refractory/resistant multiple myeloma patients treated with bortezomib. Haematologica 93: 451-454, 2008.

17. Caine GJ, Lip G, Zanetto U, Maheshwari M, Stonelake PS and Blann AD: A comparison of plasma versus histologic indices of angiogenic markers in breast cancer. Appl Immunohistochem Mol Morphol 15: 382-388, 2007.

18. Sie M, Wagemakers M, Molema G, Mooij JJ, de Bont ES and den Dunnen WF: The angiopoietin 1/angiopoietin 2 balance as a prognostic marker in primary glioblastoma multiforme. J Neurosurg 110: 147-155, 2009.

19. Brown JL, Cao ZA, Pinzon-Ortiz M, et al: A human monoclonal anti-ANG2 antibody leads to broad antitumor activity in combination with VEGF inhibitors and chemotherapy agents in preclinical models. Mol Cancer Ther 9: 145-156, 2010.

20. Dumont DJ, Yamaguchi TP, Conlon RA, Rossant J and Breitman ML: Tek, a novel tyrosine kinase gene located on mouse chromosome 4, is expressed in endothelial cells and their presumptive precursors. Oncogene 7: 1471-1480, 1992.

21. Helfrich I, Edler L, Sucker A, et al: Angiopoietin-2 levels are associated with disease progression in metastatic malignant melanoma. Clin Cancer Res 15: 1384-1392, 2009.

22. Sato TN, Tozawa Y, Deutsch U, et al: Distinct roles of the receptor tyrosine kinases Tie-1 and Tie-2 in blood vessel formation. Nature 376: 70-74, 1995.

23. De Palma M, Venneri MA, Galli R, et al: Tie2 identifies a hematopoietic lineage of proangiogenic monocytes required for tumor vessel formation and a mesenchymal population of pericyte progenitors. Cancer Cell 8: 211-226, 2005.

24. Gale NW, Thurston G, Hackett SF, et al: Angiopoietin-2 is required for postnatal angiogenesis and lymphatic patterning, and only the latter role is rescued by Angiopoietin-1. Dev Cell 3: 411-423, 2002.
25. Liang WC, Wu X, Peale RV, et al: Cross-species vascular endothelial growth factor (VEGF)-blocking antibodies completely inhibit the growth of human tumor xenografts and measure the contribution of stromal VEGF. J Biol Chem 281: 951-961, 2006

26. Thomas M and Augustin HG: The role of the Angiopoietins in vascular morphogenesis. Angiogenesis 12: 125-137, 2009.

27. Park JH, Park KJ, Kim YS, et al: Serum angiopoietin-2 as a clinical marker for lung cancer. Chest 132: 200-206, 2007.

28. Huang H, Bhat A, Woodnutt G and Lappe R: Targeting the ANGPT-TIE2 pathway in malignancy. Nat Rev Cancer 10: $575-585,2010$.

29. Nasarre $\mathrm{P}$, Thomas M, Kruse $\mathrm{K}$, et al: Host-derived angiopoietin-2 affects early stages of tumor development and vessel maturation but is dispensable for later stages of tumor growth. Cancer Res 69 : 1324-1333, 2009.

30. Mazzieri R, Pucci F, Moi D, et al: Targeting Ang2/Tie2 axis inhibits tumor growth and metastasis by impairing angiogensis and disabling rebounds of proangiogenic myeloid cells. Cancer Cell 19: 512-526, 2011

31. Bergers G, Brekken R, McMahon G, et al: Matrix mettaloproteinase-9 triggers the angiogenic switch during carcinogenesis. Nat Cell Biol 2: 737-744, 2000.

32. Bergers G and Hanahan D: Modes of resistance to anti-angiogenic therapy. Nat Rev Cancer 8: 592-603, 2008.

33. Paez-Ribes M, Allen E, Hudock J, et al: Antiangiogenic therapy elicits malignant progression of tumor and increased local invasion and distant metastasis. Cancer Cell 15: 220-231, 2009.

34. Herbst RS, Hong D, Chap L, et al: Safety, pharmacokinetics, and antitumor activity of AMG 386, a selective angiopoietin inhibitor, in adult patients with advanced solid tumors. J Clin Oncol 27: 3557-3565, 2009.

35. Rosen L, Mendelson D, Cohen R, et al: First-in-human doseescalation safety and PK trial of a novel intravenous humanized monoclonal CovX body inhibiting angiopoietin 2. J Clin Oncol 28 (Suppl 15): 2524, 2010.

36. Falcon BL, Hashizume H, Koumoutsakos P, et al: Contrasting actions of selective inhibitors of angiopoietin-1 and angiopoietin-2 on the normalization of tumor blood vessels. Am J Pathol 175: 2159-2170, 2009

37. Hashizume H, Falcon BL, Kuroda T, et al: Complementary actions of inhibitors of angiopoietin-2 and VEGF on tumor angiogenesis and growth. Cancer Res 70: 2213-2223, 2010.

38. Bagri A, Berry L, Gunter B, et al: Effects of Anti-VEGF treatment duration on tumor growth, tumor regrowth and treatment efficacy. Clin Cancer Res 16: 3887-3900, 2010

39. Ebos JM, Lee CR, Cruz-Munoz W, Bjarnason GA, Christensen JG and Kerbel RS: Accelerated metastasis after short-term treatment with a potent inhibitor of tumor angiogenesis. Cancer Cell 15: 232-239, 2009

40. Holopainen T, Saharinen P, D'Amico, et al: Angiopoietin-2 blocking antibody acts at endothelial cell-cell junctions and inhibits lung metastasis. J Natl Cancer Inst: doi: 10.1093/jnci/djs009.

41. Imanishi Y, Hu B, Jarzynka MJ, et al: Angiopoietin-2 stimulates breast cancer metastasis through the alpha(5)beta(1) integrinmediated pathway. Cancer Res 67: 4254-4263, 2007.

42. Hurwitz H, Fehrenbacher L, Novotny W, et al: Bevacizumab plus irinotecan, fluorouracil, and leucovorin for metastatic colorectal cancer. N Engl J Med 350: 2335-2342, 2004.

43. Jain RK, Duda DG, Clark JW and Loeffler JS: Lessons from phase III clinical trials on anti-VEGF therapy for cancer. Nat Clin Pract Oncol 3: 24-40, 2006.

44. Chae SS, Kamoun WS, Farrar CT, et al: Angiopoietin-2 interferes with anti-VEGFR2- induced vessel normalization and survival benefit in mice bearing gliomas. Clin Cancer Res 16: 3618-3627, 2010.

45. Koh YJ, Kim HZ, Hwang SI, et al: Double antiangiogenic protein, DAAP, targeting VEGF-A and angiopoietins in tumor angiogenesis, metastasis and vascular leakage. Cancer Cell 18 171-184, 2010. 